\title{
Effects of RF Radiation Emitted from Cellphones on Human Eye Function (Vision Acuity/Refraction)
}

Siddig Tawer Kafi, Abdalfattah Mohammed Ahmed, Bashir Algaily Ismail, Enas Ali Nayel, Asma Rashied Awad and Esraa Alzobair Alhassan

Department of Medical Physics\& Biophysics, Faculty of Science and Technology, Al-Neelain University, Khartoum 11121, Sudan

\begin{abstract}
We report on the effects of RF (radiofrequency) radiation emitted by cellphones, on the efficiency of human eyes as affected by the thermal effects of this low frequency electromagnetic radiation. A number of 68 volunteer students from the faculty of optometry at Al-Neelain University, Khartoum, Sudan, were randomly selected for the purpose of this study. The ages of tested students ranged between 18 and 25 years, none of which complain of sight complications (vision or refraction). A questionnaire combined with vision and refraction tests was applied. It was found that apart from which side of the head cellphone was used, the majority (80\%) complain no problems with vision acuity test, but in the contrary approximately $90 \%$ showed to complain from defects in refraction for both eyes. It was concluded that defects in the refraction of human eyes could be attributed to thermal effects of RF radiation emitted from cellphone during phone calls. Reduction of call duration when using cellphone in direct contact with the head was recommended.
\end{abstract}

Key words: Cellphone, RF radiation, human eye, vision acuity/refraction.

\section{Introduction}

RF (radiofrequency) waves characterized by their frequency, intensity of electric and magnetic fields, direction and polarization, are non-ionizing radiation with wavelengths longer than infrared radiation. They are used for mobile communication, broadcasting, radar, satellite communication, computer networks and other applications.

Weak electromagnetic fields such as RF radiation emitted from mobile phones interact with the human body and affect the human tissues $[1,2]$. Because the nature of the interaction between biological tissue and $\mathrm{RF}$ radiation is complex, the SAR (specific absorption rate) defined as the rate of RF radiation power absorbed per unit mass by any part of the body (in W/Kg), is used to measure the energy dose of RF radiation absorbed. SAR value depends on various factors such as: the nature of field (low or high frequency); distance and spatial orientation of the field relative to an object; object's geometry, and water content of tissues. SAR

Corresponding author: Siddig Tawer Kafi, Ph.D., assoc. prof., research field: biophysics. also varies according to the handset model, the transmission system technology (analog or digital), the distance between a user's head and the handset antenna, and the distance from nearest base station [3].

Effects of RF and MW (microwave) radiation on biological tissues are mainly classified into thermal and non-thermal effects. The tissue temperature increase resulting from exposure to EMW (electromagnetic waves) is referred to as "thermal effects". Heat generation depends on SAR value and power density of the emitted radiation [4]. These effects may cause disruption of cell function and development. A half hour exposure to high frequency, low intensity field within the SAR range of $1 \mathrm{~W} / \mathrm{kg}$ to $4 \mathrm{~W} / \mathrm{kg}$ body weight can raise temperature up to the $1{ }^{\circ} \mathrm{C}$ in adult healthy people $[5,6]$.

Due to the drastic growth of wireless telecom and the wide use of cellphones worldwide, there has been important need for research effort to answer the question of safety and health effects of this emerging technology. During calls the most probable parts in human head that would be affected by exposure to $\mathrm{RF} / \mathrm{MW}$ radiation are the ear, eye and brain. From 
previous studies concerning the effect of RF/MW emitted from cellphones, Kojima et al. [7] studied the thermal effects on rabbit's eyes exposed to microwave. One of the rabbit eyes was exposed to $2.45 \mathrm{GHz}$ for 20-60 $\mathrm{min}(180 \mathrm{~W} / \mathrm{kg})$. The results showed meiosis, conjunctiva congestion, corneal edema, increased light scattering of anterior shallow cortex in papillary area of lens. Loughran et al. [8] investigated the effects on human sleep. They indicated that mobile phone exposure prior to sleep may promote rapid eye movement and modify the sleep electroencephalogram in the first non-rapid eye movement sleep period. Due to Yao and his group electromagnetic noise could increase cultured HLECs (human lens epithelial cells) upon exposure to $1.8 \mathrm{GHz}$ [9]. In addition, Demiral et al. [10] in 2012 studied the effect of EM radiation emitted by 3G (third generation) mobile phones on anti-oxidation and oxidative stress parameters in eye tissue and blood of rats. Buccella and coworkers [11] studied the effects of different RF sources on the SAR (specific absorption rate) and maximum temperature increase in the human eye using a new model of the human head and compared with an anatomical model of the visible human.

The main objective of this work was to study the effects of RF emitted from cellphones on human eye function, specifically vision acuity and refraction. Extended call periods may increase the temperature of the user's eye tissues and influence its natural functioning.

\section{Methodology}

A questionnaire included age, sex, period of time using the phone, side of head mostly used during calls, and the type of phone used, was designed and filled by 68 adult volunteers who were randomly selected for the purpose of this study. Their ages ranged between 18 and 25 years, and they all complain no historical eye disease. The cell phones used during the period of this study were of the type $3 \mathrm{G}$. The minimum calls duration per day ranges from one minute to one hour for the selected volunteers.

This scientific study is a double-blind and randomized to ensure that the study conducted without bias.

Vision acuity and refractive error tests were conducted for all volunteers. Vision acuity was done in each eye separately. It was determined by the size of letters.

Refractive error test determines how lenses focus light perfectly. Retinoscopy technique was used to obtain an objective measurement of the refractive error of the eye. The refractive error determined according to the movement of light with lenses axis (vertical/horizontal). The examiner uses to shine light into the eye and observed the reflection off the retina. If it is in the same direction as the axis, this had two probabilities: normal or hyperopia and they were distinguished by 1.5 lenses. If it is normal with the axis, then reflection value equals zero. For the hyperopia it is corrected by positive lens with certain value chosen from a "trail set" which contained several correction lenses with different values. If the movement of light with the lens axis was against then it would be diagnosed as myopia and this is corrected by negative lens value (i.e., -0.5). Data were then collected and tabulated. Excel computer program version (2010) was used for the analysis of the obtainable data.

\section{Results and Discussion}

Results obtained during this work are summarized in Figs. 1-5 below. They show the output of both vision and refraction tests. Generally most of the cellphone users use cellphones in contact to the right side of the head, which means that this side is exposed during calls more than the left side. Apart from this $80 \%$ were found to be of normal vision for both eyes, while only $11.7 \%$ complain abnormality of vision acuity for both eyes as shown in Fig.1. In the contrary approximately $90 \%$ of the cellphone users showed defects in the refraction test for both eyes, and only 3\% complain defects in a single eye while the rest showed normal refraction. 


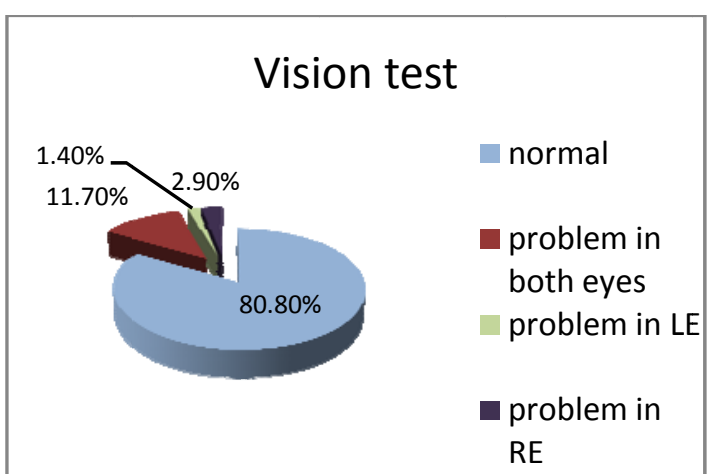

Fig. $1 \%$ of normal and abnormal vision acuity for both eyes. LE: left eye and RE: right eye.

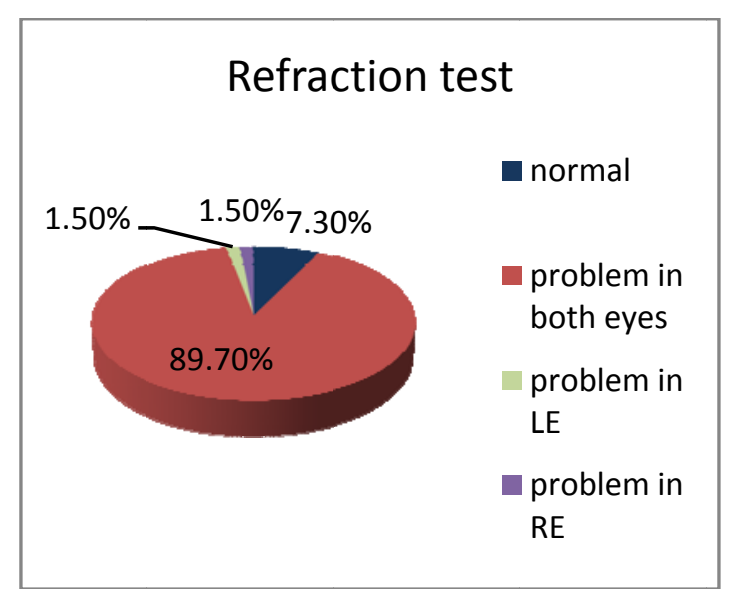

Fig. 2 \% of normal and abnormal refraction for both eyes, LE and RE as in Fig. 1.

Refraction defects could be due to deformation in the tissues of the eye, due to thermal effects of RF radiation during phone calls. As a consequence a shift in the axis of the eye occurs, leading to refraction abnormality.

When we consider the side of the head used during calls, it came out that almost the same result was obtainable for refraction apart from the side used. The majority complain refraction defects in both eyes. Fig. 4 (a \& b) shows detailed values of refraction for the right side users. Fig. 4. (a): gives values for the left eye and (b) for the right eye. Values are fluctuating with no consistency between left and right eye. The same result was obtainable for the volunteers who used the left side of the head as Fig. 5 (a \& b) shows. But generally both figures confirm defects in the refraction for those who use the left or right side of the head for phone calling. This could be attributed to transfer of heat energy dumped from the cellphone as RF by the regulatory system of the human body. That is to say the exposure of the eye which is closer to the side of cellphone used, may lead to increasing the temperature of the eye's tissues, but some of this heat energy will reach the second eye by heat transfer mechanism and consequently rise its temperature. As long as a user continues using the cellphone both left and right eye are subject to thermal effect from the RF leading to deformation in both eyes and hence altering their axis of refraction.

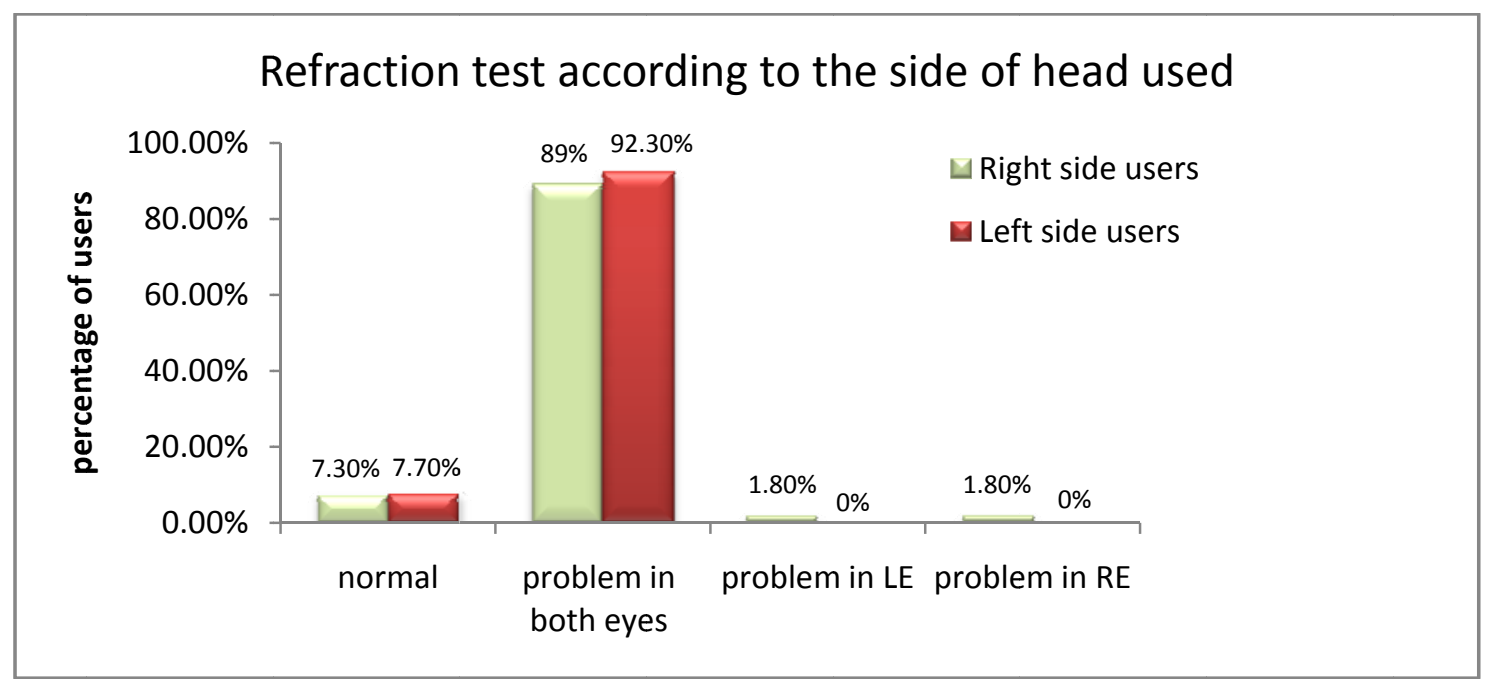

Fig. $3 \%$ of normal and abnormal refraction for both eyes, LE and RE for left and right eye respectively. 

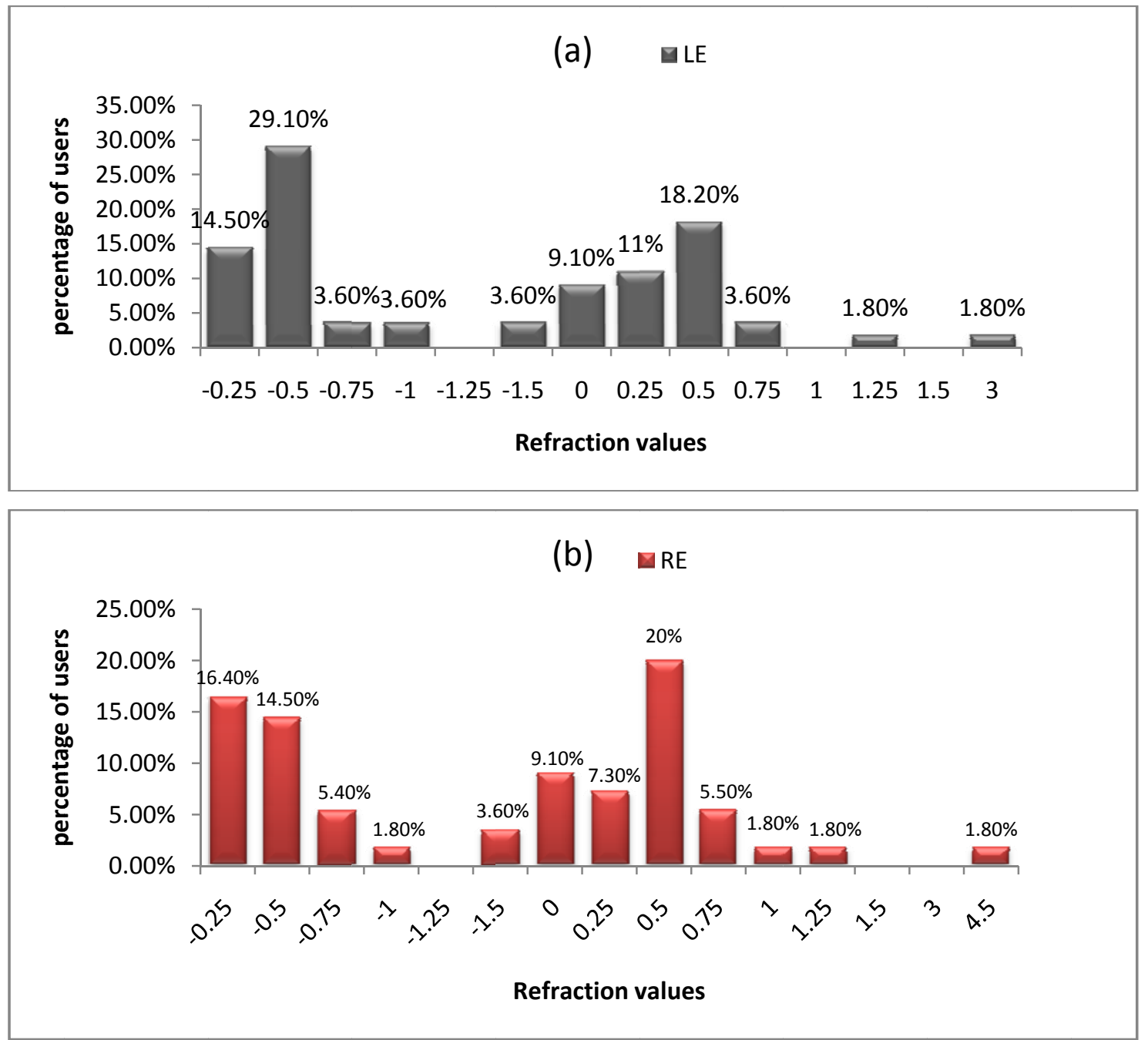

Fig. 4 Detailed valuesof refraction test for right side users. (a): left eye; (b): right eye.

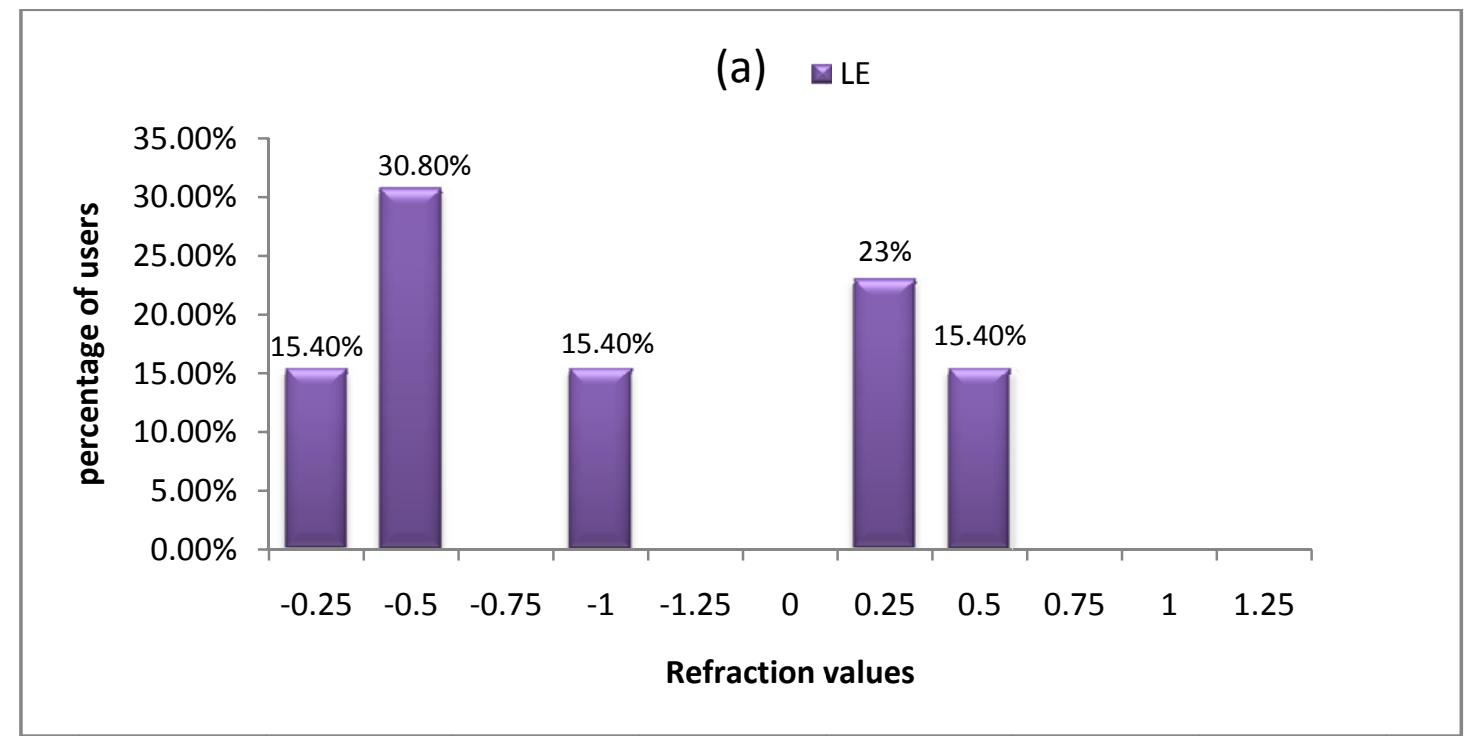




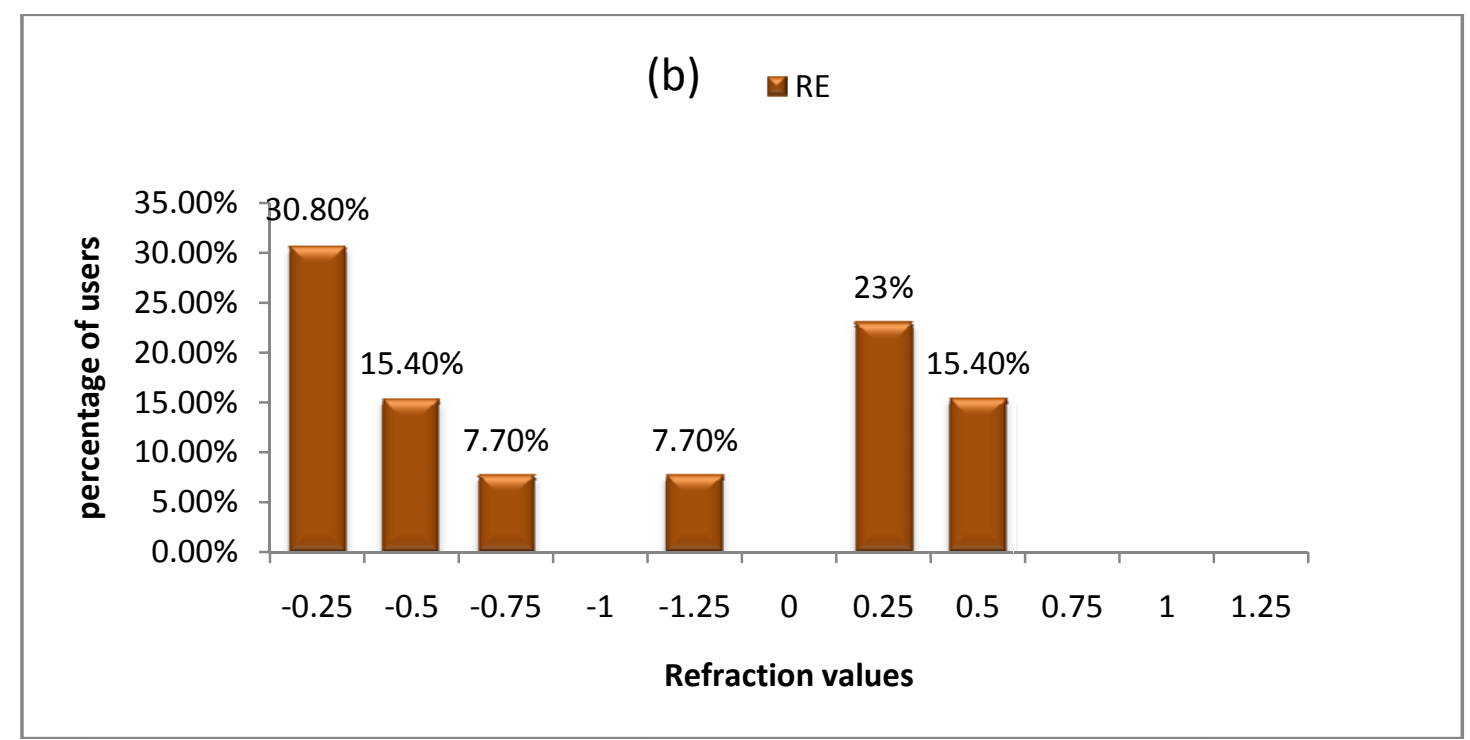

Fig. 5 Detailed values of refraction test for left side users. (a): left eye; (b): right eye.

\section{Conclusions}

The influence of cellular phones on human eyes based on the thermal effects of RF radiation was studied. Although vision defects recorded were marginal, refraction defects were found to be dominant regardless of which side of the head was used (i.e., right or left). Wider spectrum and larger number of volunteers are needed for comprehensive study to clarify the correlation between thermal effects of RF radiation from cellphones and human sight defects. This study was restricted to $3 \mathrm{G}$ generation of cellphones only, therefore adding $4 \mathrm{G}$ generation in the future study is needed to figure out the relation between phone generation and the effects on human eye function if any. Choosing good quality cellphone with minimum SAR value in addition to reduction of call duration is strongly recommended.

\section{Acknowledgement}

The authors would like to thank the faculty of optometry at Al-Neelain University for technical support of this work.

\section{References}

[1] Blank, M. (Ed.). 1995. Electromagnetic Fields: Biological Interactions and Mechanisms. American Chemical Society, Book 250, Columbia University.
[2] Balbani, A. P., and Montovani, J. C. 2008. "Mobile Phones: Influence on Auditory and Vestibular Systems.” Bra.z J. Otorhinolaryngol 74: 125-31.

[3] Bhat, M. A., and Kumar, V. 2013. "Calculation of SAR and Measurement of Temperature Change of Human Head Due to the Mobile Phone Waves at Frequencies $900 \mathrm{MHz}$ and 1800 MHz." Advances in Physics Theories and Applications 16: 54-63.

[4] Buccella, C., De Santis, V., and Feliziani, M. 2007. "Prediction of Temperature Increase in Human Eyes Due to RF Sources." IEEE Transactions on Electromagnetic Compatibility 49: 825-33.

[5] Tesylier, G., Tonduli, L., Malabiau, R., and Debouzy, J. C. 2002. "Effects of Exposure to Level Radiofrequency Fields on Acetylcholine Release in Hippocampus of Freely Moving Rats.” Bioelectromagnetics 23: 249-55.

[6] Balci, M., Devrim, E., and Durak, I. 2007. "Effects of Mobile Phones on Oxidant/Antioxidant Balance in Cornea and Lens of Rats.” Curr. Eye Res. 32: 21-5.

[7] Kojima, M., Hata, I., Wake, K., Watanabe, S., Yamanaka, Y., Kamimura, Y., Taki, M., and Sasaki, K. 2004. "Influence of Anesthesia on Ocular Effects and Temperature in Rabbit Eyes Exposed to Microwaves.” Bioelectromagnetics 25: 228-33.

[8] Loughran, S. P., Wood, A. W., Barton, J. M., Croft, R. J., Thompson, B., and Stough, C. 2005. "The Effect of Electromagnetic Fields Emitted by Mobile Phones on Human Sleep.” NeuroReport 16: 1973-6.

[9] Yao, K., Wu, W., Wang, K., Ni, S., Ye, P., Yu, Y., Ye, J., and Sun, L. 2008. "Electromagnetic Noise Inhibits Radiofrequency Radiation-Induced DNA Damage and Reactive Oxygen Species Increase in Human Lens Epithelial Cells.” Mol. Vis. 14: 964-9.

[10] Demirel, S., Doganay, S., Turkoz, Y., Dogan, Z., Turan, 
B., and Firat, P. G. 2012. "Effects of Third Generation Mobile Phone-Emitted Electromagnetic Radiation on oxidative Stress Parameters in Eye Tissue and Blood of Rats." Cutan Ocul Toxicol 31: 89-94.
[11] Buccela, C., De Santis, V., and Feliziani, M. 2007. "Prediction of Temperature Increase in Human Eyes Due to RF Sources.” IEEE Transactions on Electromagnetic Compatibility 49: 825-33. 